\title{
Chapter 12 \\ Laboratories for Research on Freight Systems and Planning
}

\author{
André Romano Alho, Takanori Sakai, Fang Zhao, Linlin You, Peiyu Jing, \\ Lynette Cheah, Christopher Zegras, and Moshe Ben-Akiva
}

\begin{abstract}
Advancements in information and communication technologies (ICT) and the advent of novel mobility solutions have brought about drastic changes in the urban mobility environment. Pervasive ICT devices acquire new sources of data that can inform detailed transportation simulation models, and are useful in analyzing new policies and technologies. In this context, we developed software laboratories that leverage the latest technological developments and enhance freight research. Future mobility sensing (FMS) is a data-collection platform that integrates tracking devices and mobile apps, a backend with machine-learning technologies and user interfaces to deliver highly accurate and detailed mobility data. The second platform, SimMobility, is an open-source, agent-based urban simulation platform which replicates urban passenger and goods movements in a fully disaggregated manner. The two
\end{abstract}

A. R. Alho $(\bowtie) \cdot$ T. Sakai · F. Zhao

Future Urban Mobility Interdisciplinary Research Group, Singapore-MIT Alliance for Research and Technology, Queenstown, Singapore

e-mail: andre.romano@smart.mit.edu

L. You

School of Intelligent Systems Engineering, Sun Yat-sen University, Guangzhou, China

e-mail: lyou@mail.sysu.edu.cn

P. Jing

Intelligent Transportation Systems Lab, Massachusetts Institute of Technology, Cambridge, USA e-mail: peiyu@mit.edu

L. Cheah

Department of Engineering Systems and Design, Singapore University of Technology and Design, Tampines, Singapore

e-mail: lynette@ sutd.edu.sg

C. Zegras

Department of Urban Studies and Planning, Massachusetts Institute of Technology, Cambridge, USA

e-mail: czegras@mit.edu

M. Ben-Akiva

Department of Civil and Environmental Engineering, Massachusetts Institute of Technology,

Cambridge, USA

e-mail: mba@mit.edu 
platforms have been used jointly to advance the state of the art in behavioral modeling for passenger and goods movements. In this chapter, we review recent developments in freight-transportation data-collection techniques, including contributions to transportation modeling, and state-of-the-art transportation models. We then introduce FMS and SimMobility and demonstrate a coordinated application using three examples. Lastly, we highlight potential innovations and future challenges in these research domains.

\subsection{Introduction}

The urban mobility system, including passenger and goods movements, is becoming more complex. Demand for mobility is growing and, at the same time, the roles to be played, modes available, and system-wide synergies are becoming more diverse. These changes have been stimulated by the evolution of information and communication technologies (ICT). For example, crowdsourcing initiatives allow individuals to become temporary freight carriers. These and other changes show a clear need for simulation tools that allow researchers, industry practitioners, and urban planners to better grasp the potential impacts of technologies and policies in the urban mobility system. Despite their predominantly passenger-centric development, stateof-the-art behavioral simulation models are now capable of replicating business-tobusiness transactions between agents that can play multiple roles (shipper, carrier, and receiver) in a disaggregate manner. The next generation of models is expected to extend its capabilities to cover business-to-consumer and consumer-to-consumer flows, which are becoming more important as e-commerce plays a larger role in urban goods movements. Moreover, as the boundaries between passenger and goods movements become dimmer, new challenges to the development of integrated models will arise. The increasing ability to comprehensively represent relevant agents' decisions and behaviors is associated with a need for fine-resolution data. Still, data collection for freight remains a challenge, plagued by low participation rates for surveys and hard-to-reach key respondents. Innovations in the methods for collecting freighttransportation data are sought, leading to expectations of relying on sensing technologies and Big Data sources to overcome the data limitations. At this point in time, these new sources of data are minimally incorporated into transportation models for testing a wide range of policies and technologies.

This chapter consists of four sections, presenting (1) future mobility sensing (FMS), a freight data-collection platform, (2) SimMobility, an urban land-use and transport-simulation platform, and (3) examples of their coordinated use to move forward the current domain knowledge. The first two sections start with self-contained literature reviews on relevant research, including basic techniques, methods and applications. They are followed by a detailed account of the laboratories, FMS and SimMobility, as well as past and current applications. In Sect. 12.4, we provide examples of the coordinated use of the laboratories, and finally, we conclude with a summary and future research directions in Sect. 12.5. 


\subsection{Future Mobility Sensing, a Behavioral Laboratory}

\subsubsection{Background}

The practice of transportation modeling and planning relies on a variety of data for both passenger and goods movements. Particularly for freight-transportation, highquality data is required for the development of simulation models for commodity flows and freight-vehicle operations. Data-collection efforts in the urban freight domain need to deal with a variety of agents (e.g., companies, establishments, and vehicle drivers) in terms of decision-making mechanisms and behaviors. The heterogeneity of agents and agent types makes it challenging, compared to passenger movements, to collect a comprehensive dataset that portrays their joint decisions. As a result, multiple data-collection approaches are used which, in broad terms, can be categorized into four main groups.

\subsubsection{Static and Count Data}

These are data collected through fixed location sensors such as inductive loop detectors, automatic vehicle classifier systems, weight-in-motion (WIM) systems, or video systems. Although road-based sensors, such as inductive loop detectors, are inherently limited to capture fine-resolution freight counts, Tok (2008) developed a highfidelity inductive loop sensor to achieve commercial vehicle classification based on the inductive signatures of vehicle types, demonstrating their potential to provide information-rich commercial vehicle traffic-count data.

The installation of video cameras made traffic counts easier than in the past, particularly for congested settings or when attempting to disaggregate the data by vehicle types. Zhang et al. (2007) detailed a video-based vehicle detection and classification (VVDC) system for collecting vehicle count and classification data using uncalibrated video images. The proposed approach was demonstrated with high accuracy, although there are a series of enhancements suggested to deal with longitudinal vehicle occlusions, severe camera vibrations, and headlight reflection problems. Mammes and Klatsky (2017) presented a video-based system to assess freight loading-bay demand and availability. Sun et al. (2017) have used video cameras for monitoring local freight traffic movements with fine resolution by developing computer-vision algorithms.

\subsubsection{Dynamic and Mobile Data}

These are data collected through sensors that move with vehicles, using devices such as GNSS, on-board diagnostics (OBD), or similar telematics. GPS data are often collected by companies for monitoring their vehicles. One of the most widely known truck GPS datasets is published by the American Transportation Research Institute 
(ATRI). This dataset considerably contributes to freight research in the USA and has been used for multiple purposes, including the development of truck route-choice data (Kamali 2015) and the generation of statewide freight-truck flows (Zanjani 2014). It is often fused with other datasets because, despite its large size, it lacks details on commodities carried or trip purposes (Eluru et al. 2018). An alternative to data fusion is to complement GPS tracking with surveys, which will be discussed later in this chapter.

\subsubsection{Survey Data}

Data can also be collected through surveys that target drivers, fleet managers, or warehouse employees, among others. There are various designs of freight surveys. Freight survey design and its applications are summarized by Allen et al. (2012), covering establishment surveys, vehicle observation surveys, parking surveys, driver surveys, commodity-flow surveys, roadside-interview surveys, and other surveys. Cheah et al. (2017) provided a literature review focused on commodity and establishment-based freight surveys.

\subsubsection{Indirect Data}

This refers to data from sources that are not designed to inform freight models or derive freight-related insights, but could be used for such purposes. Some sources of Big Data would fit this category.

A challenge for freight-transportation data collection is that a single method only allows for a partial view of the urban freight distribution system, as indicated by Holguín-Veras and Jaller (2013). The same authors also detailed the strengths and weaknesses of several of the data-collection methods. Some of the above-mentioned surveys have leveraged novel technologies, although not to a great extent. Despite a greater number of freight data-collection efforts taking place, several surveys are still paper-based, although Web-based surveys reduce the burden of data entry and associated errors and are becoming more common (e.g., the Lisbon Establishment-based Freight survey described by Alho and de Abreu e Silva 2015). A major challenge lies in the fact that user-reported data are prone to inaccuracies as respondents often need to recall past activities. Furthermore, the aforementioned high-resolution data needed for modeling and simulation purposes can easily lead to extensive surveys which respondents might not be willing to fill in. Jeong et al. (2016) highlighted the challenges of ensuring sufficient participation to achieve a meaningful sample size, based on the experience of pairing a Web-based fleet manager survey and a smartphone app-based driver survey to pilot a preliminary design for the California Vehicle Inventory and Use Survey (CAL-VIUS).

In summary, we found three main research thrusts in freight data collection that call for greater attention. First, the innovative use of technology, including sensing technologies, as a means to reduce user burden requires further advances. Second, as 
it is challenging to recruit participants for freight surveys, there is a need to design incentive methods that can effectively increase response rate and encourage long-term participation. Some of these efforts have been piloted in household travel surveys (Nahmias-Biran et al. 2018) and are related to informational incentives, which can complement or be alternatives to monetary incentives. Third, new and alternative data sources have to be explored. Ludlow and Sakhrani (2017), present a report (NCFRP 49-New Source of Freight Data for Urban and Metropolitan Mobility) that focuses on new data sources to address urban and metropolitan freight challenges. The highlighted novel and potentially useful data sources include crowdsourced data, road and vehicle sensors (Bluetooth, RFID, connected vehicles), vehicle data streams, or image data (such as satellite-based). The FMS platform aims to address these three research areas and is a flexible and comprehensive behavioral laboratory for freight data collection.

\subsubsection{FMS Architecture}

Future mobility sensing (FMS) is a data-collection and visualization platform that leverages mobile sensing technology, machine-learning algorithms, and user verification to provide details of mobility behavior of passengers or freight. It was first developed as a smartphone-based automated household travel survey system. In a second iteration, it was extended to support commodity-flow surveys and track freight and commercial vehicles (FMS-Freight). FMS-Freight collects and processes survey data from business establishments related to the role(s) they play in goods movements (shipping, receiving, and transporting), associated shipments, and vehicle operations, and it also collects trip information from the drivers. FMS consists of the three distinct but interconnected components illustrated in Fig. 12.1:

- A mobile app and tracking devices that leverage various sensing technologies;
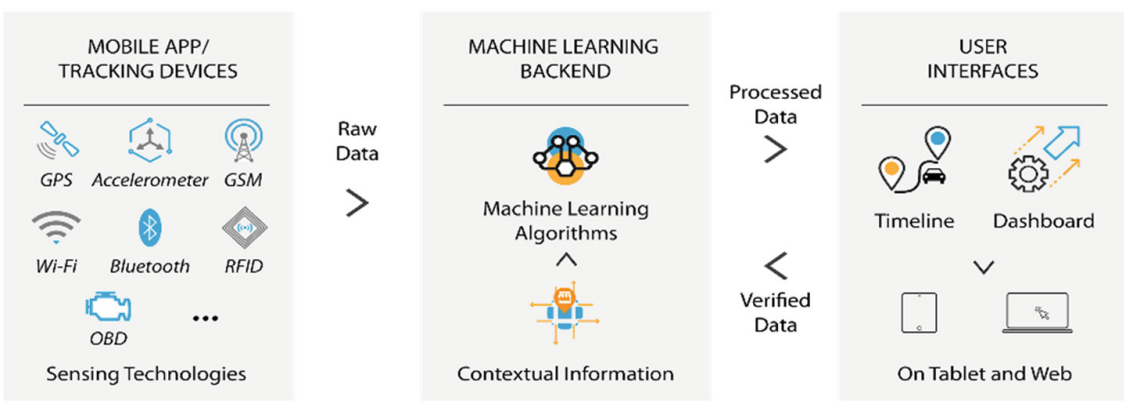

Fig. 12.1 Future mobility sensing (FMS) platform architecture 
- A backend consisting of a server system with (a) a database and (b) custom algorithms to infer stops, trip purposes, and other trip details, to reduce user burden; and

- User interfaces, both mobile and Web-based, used for verification of activities by respondents and displaying summarized information (e.g., a dashboard as described by You et al. 2018).

When FMS is used to support freight data collection, the details of each component are as follows.

\subsubsection{Mobile App/Tracking Devices}

FMS-Freight supports the collection of raw data from various mobile sensing devices, such as tablets, GPS loggers, and OBD devices. GPS loggers and OBD devices are primary tools to collect data. Data are gathered from several sensors and uploaded to the backend for analysis. These devices can be easily installed and attached, respectively, to vehicles and shipments, and can collect location information with high accuracy. In the case of collecting vehicle trajectory data, the use of the vehicle battery to power the device allows for uninterrupted multi-day data collection.

\subsubsection{Backend}

Backend machine-learning algorithms process collected raw data together with the user-verified timeline (i.e., records of activities, verified through user interfaces detailed below) and contextual information (e.g., POI data) to infer stops and stop activities (Zhao et al. 2015). For shipment tracking, travel modes are also detected, which can be used to further reduce the user's verification burden. Verified data are fused and post-processed to support the identification of vehicle and shipment patterns.

\subsubsection{User Interfaces}

User-friendly interfaces on both tablet and Web applications allow a user to review and verify her or his timeline and activities. Daily verification includes confirming inferred information and filling missing information (i.e., activities, commodity type) as illustrated in Fig. 12.2. The data verified by the user are subsequently used to further train the algorithms for inferences. Moreover, the interface allows for the generation of a summary of activities in a dashboard for a user to review. An example of a shipment trace is presented in Fig. 12.3. 


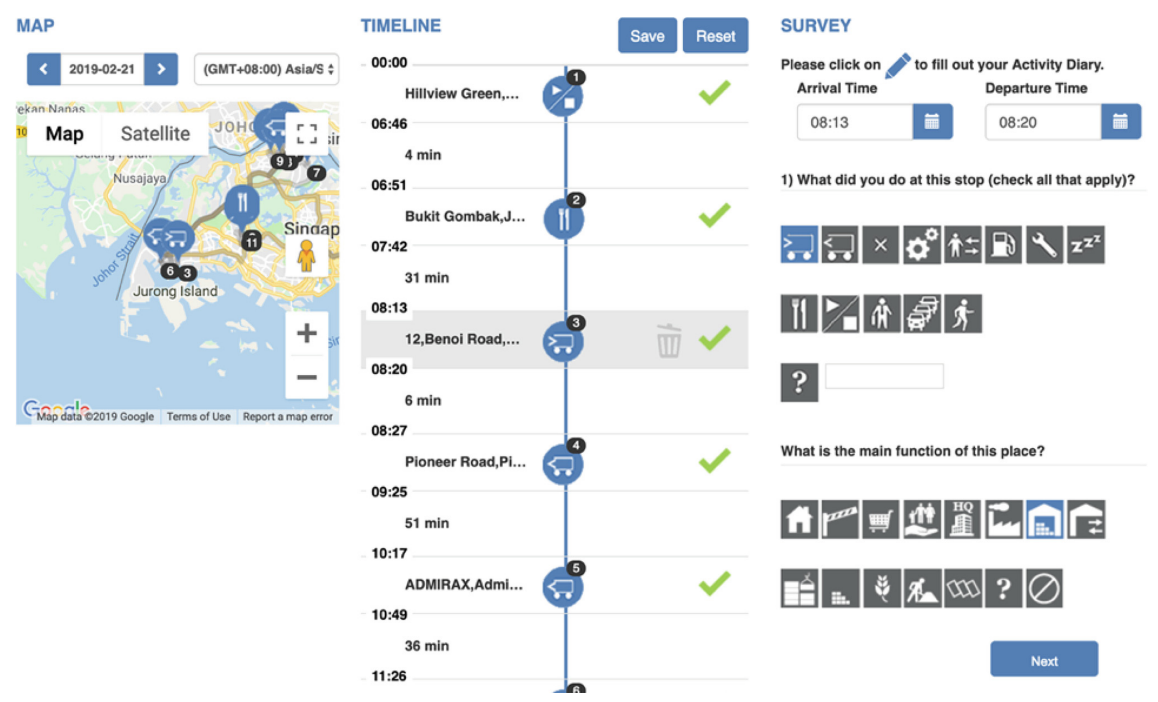

Fig. 12.2 FMS-freight stop verification interface for drivers

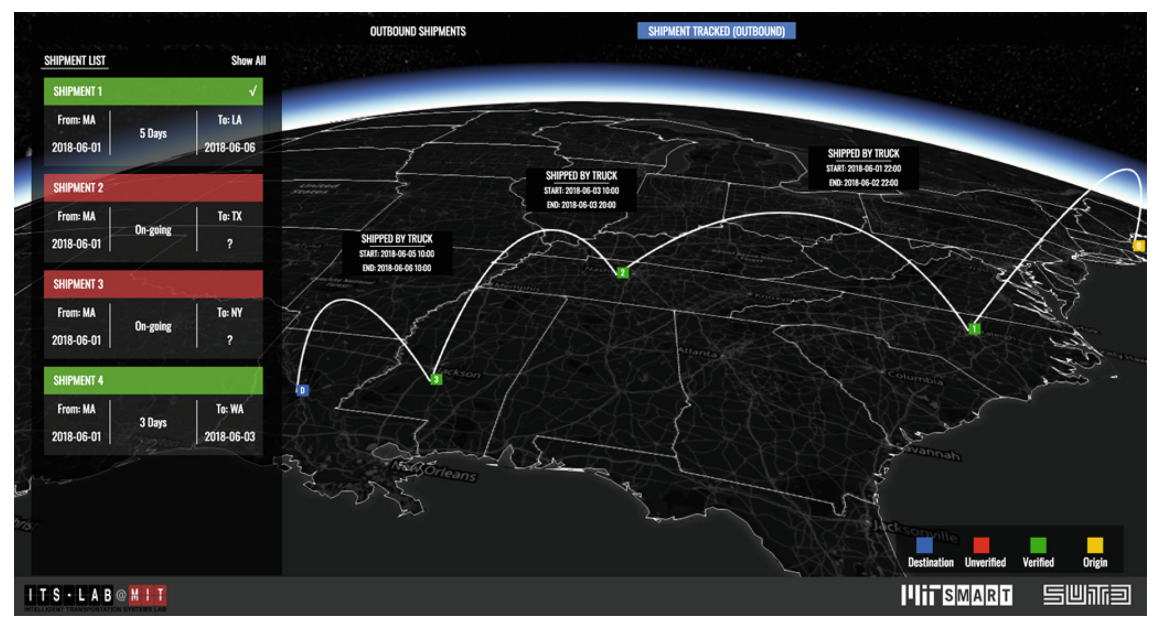

Fig. 12.3 Shipment dashboard, a form of informational incentive

\subsubsection{Applications}

FMS-Freight can be used to support applications ranging from truck-driver surveys, shipment-tracking surveys, or full-fledged integrated commodity-flow surveys (CFS). The survey process for integrated CFS is shown in Fig. 12.4, which consists of three steps: first, registration and pre-survey for establishment and driver information; second, shipment and freight-vehicle tracking; and lastly verification of 


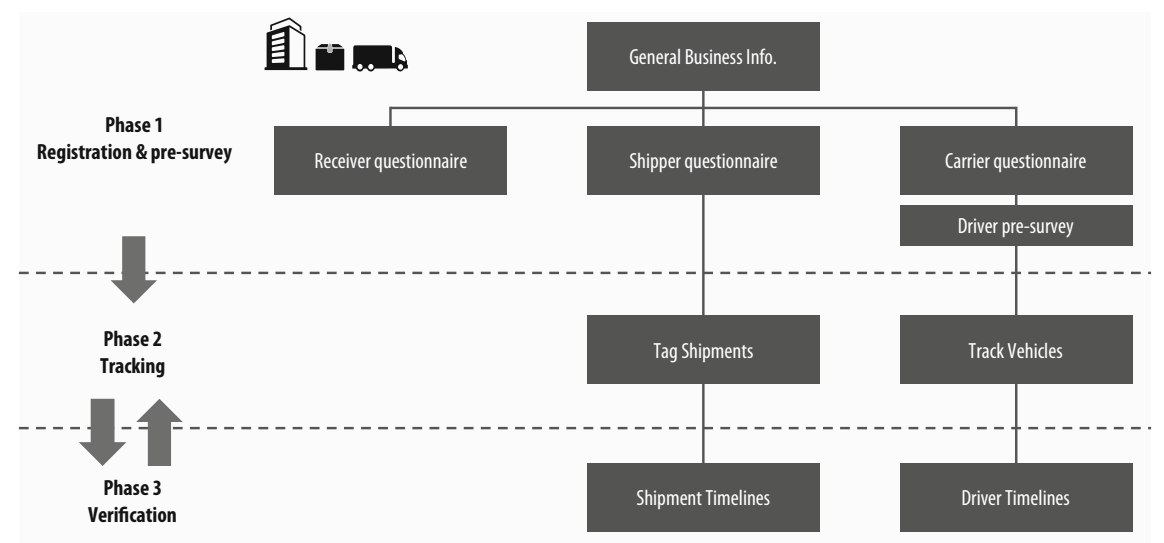

Fig. 12.4 Integrated commodity-flow survey process

inferred activities based on the tracking data. The tracking and verification steps are an iterative process that can span days or weeks depending on the survey needs.

While being continuously developed and enhanced, the FMS-Freight platform has so far been employed in the following pilots:

- A GPS-based inter-city truck-driver survey, which includes tracking, verification and stated preferences survey on driver routing behavior (Ben-Akiva et al. 2016).

- A large-scale GPS-based vehicle tracking and driver-activity survey to a sample of season parking ticket holders of the Urban Redevelopment Authority heavy vehicle parks to understand movements and parking patterns (Alho et al. 2018).

- A pilot of a commodity-flow survey in Singapore (Cheah et al. 2017), which is being followed by a larger deployment, to understand commodity flows and associated business characteristics.

- A shipment-tracking pilot in the USA and Singapore to gain additional understanding of the supply chain structures that shipments go through.

\subsection{SimMobility, a Simulation Laboratory}

\subsubsection{Background}

Simulation models have been developed and used to meet analytical and policy needs in city planning for decades. Regarding transportation, the models that simulate traffic flows are used to predict the future transportation environment and evaluate technology and the impacts of policy measures, providing the basis for policy decisions. With the increasing need for models that are able to handle a variety of technology 
and policy changes, the past few decades saw remarkable progress in the capability of transportation simulation tools. Classical aggregate models are being replaced with disaggregate, agent-based models. These novel simulation tools capture the complex mechanism of decisions associated with the movements of passenger and goods. As such, they enable the use of simulations to support the analysis of land-use and transportation systems changes, infrastructure management (e.g. dynamic road pricing), and emerging mobility services (e.g., shared and on-demand vehicles) among others.

The above-mentioned trend also applies to urban freight models for which advanced frameworks were proposed around 2000 and after. A number of agentbased urban freight models, which take into account behavioral mechanics in supply chain and logistics operations, have been proposed as alternatives to traditional aggregate commodity- or truck-based models (Chow et al. 2010). Those models simulate the decisions and behaviors of different agents, such as shippers, receivers, carriers (including drivers), and policymakers, and their interactions for commodity flows, logistics and transportation services, and transportation infrastructure usage (Boerkamps et al. 2000; Wisetjindawat et al. 2005; Fischer et al. 2005; Roorda et al. 2010). The resultant improvement of the granularity in decisions and behaviors allows a model to capture the inter-relations among them in a reasonable and reliable manner. The increase in data availability for specific regions and the advent of new data-science techniques further promote the development and application of disaggregate models, which, by their nature, require extensive data inputs. Thus, the potential for using them in real-world planning practices has been increasing. However, at a global level, a shortage of suitable data hampers the widespread applications of such models. In the USA, agent-based freight models were developed for some metropolitan regions, including the Chicago region (Outwater et al. 2013; RSG 2015) and the Arizona Sun Corridor Megaregion (Livshits et al. 2018). One example of this type of model is SimMobility (Adnan et al. 2016), an open-source urban simulation platform developed by the Singapore-MIT Alliance for Research and Technology (SMART) and the Intelligent Transportation Systems (ITS) Lab at Massachusetts Institute of Technology. Targeting urban freight modeling, a set of SimMobility components was estimated and calibrated for Singapore. This set of components adds the capability of simulating goods movements across supply chains, as well as agents' reactions to freight-focused policies. Examples of the latter are route restrictions, urban consolidation schemes, off-hour deliveries, and overnight, pickup, and delivery parking choices. We provide an overview of the simulation tool in this section. The details of the tool, including model specifications, are available in the paper by Sakai et al. (2019).

\subsubsection{SimMobility Architecture}

SimMobility is an agent-based simulation platform consisting of models for landuse changes and passenger and goods movements at the metropolitan scale. The simulations in SimMobility are fully disaggregated and maintain the consistency of 
agents. In SimMobility, three temporal layers are considered (Fig. 12.5): long-term (LT), mid-term (MT), and short-term (ST). The LT model covers the components of urban simulation, such as residential and firm locations, school and work locations, vehicle ownership, and parking locations, as well as business relationships among firms. The MT model, on the other hand, simulates activities of individuals, logistics operations, and vehicle and transportation-system operations at the daily level. The short-term (ST) model is a microscopic simulator for the movements of agents within a day. The different modules share a single database which maintains the data about agents, land use, transportation, and activities, enabling data exchange across the modules. The fine-resolution simulations also allow for keeping track of the behaviors of individual agents, or of knowing specifically on which vehicle a shipment was loaded.

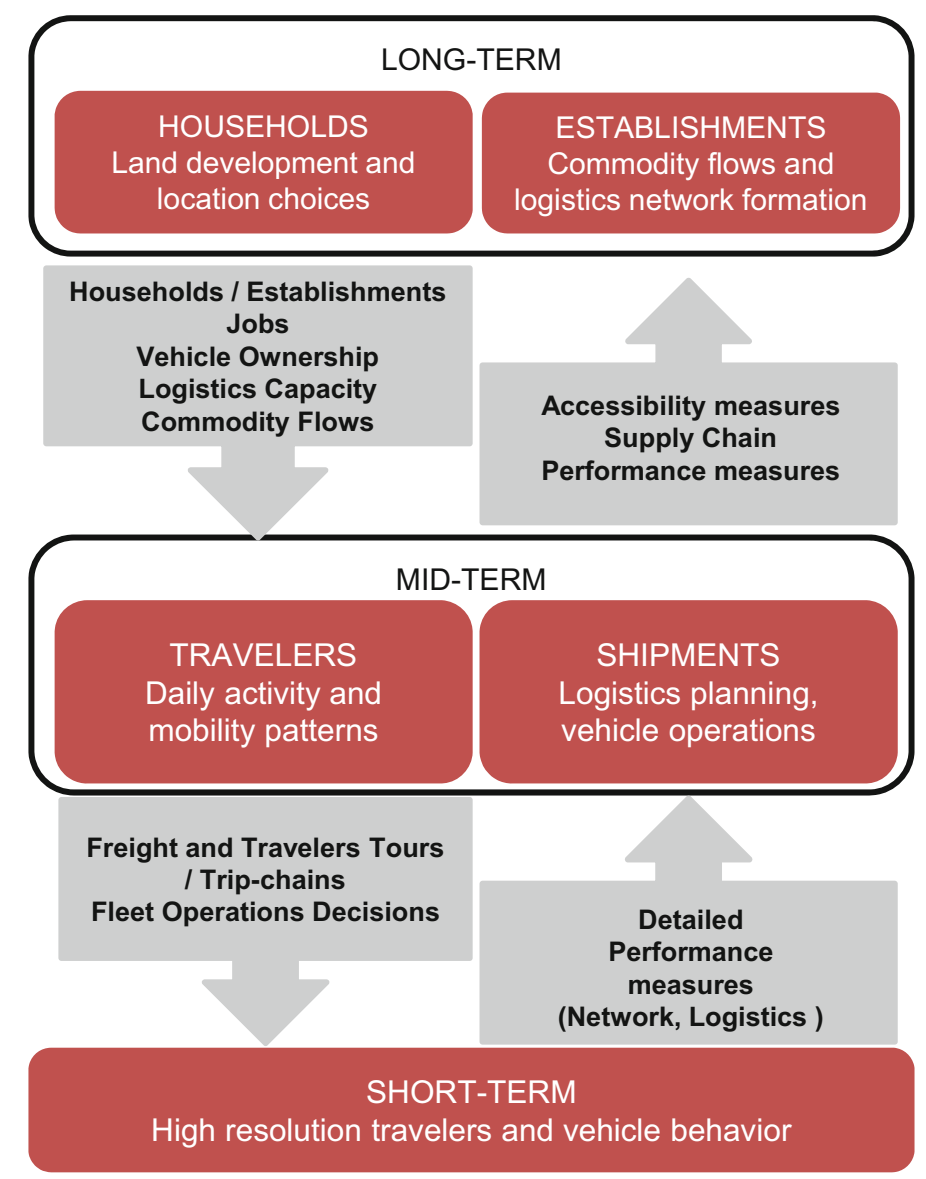

Fig. 12.5 SimMobility framework 
To date, the platform has been deployed for the Greater Boston area, the Baltimore region, and Singapore as well as several prototypical cities. The freight models are currently estimated for Singapore. Further details of different components of SimMobility are available in the literature (Adnan et al. 2016; Zhu et al. 2018; Lu et al. 2015; Azevedo et al. 2017). The models incorporated in SimMobility were developed using a variety of datasets, including those obtained from FMS.

The set of components for freight simulation, termed the freight simulator hereafter, was designed for advancing the state of the art in urban freight modeling practices. It should be noted that the freight simulator is integrated with other components in SimMobility, sharing some modules, such as micro- and meso-scale traffic simulators, as well as taking inputs with passenger simulation. Figure 12.6 shows the main modules of the freight simulator, which follow the above-mentioned three temporal layers. The LT model simulates commodity contracts, which define commodity flows (i.e., selling and purchasing policies), and overnight parking choices for freight vehicles. The MT model simulates pre-day logistics planning and within-day vehicle operations, translating commodity flows to vehicle operations and behaviors, and subsequently to transport-network conditions. Lastly, the ST model simulates the behaviors of agents at an increased level of detail, particularly regarding driver behaviors, using car-following and lane-changing models. Each module, excluding the ST model, is briefly described below. A detailed description of the ST model, the microscopic traffic simulator, is available by Azevedo et al. (2017).

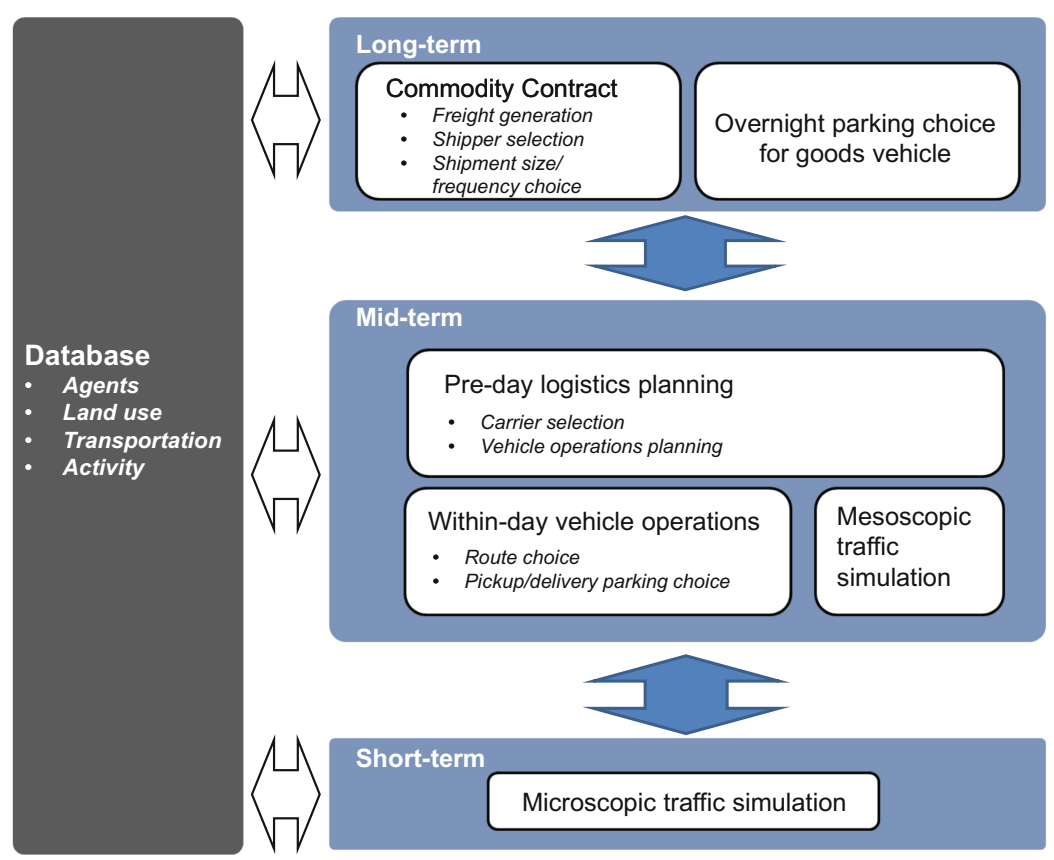

Fig. 12.6 Major components of the freight simulator 
In freight simulations, business establishments play a key role. An establishment is characterized by location, employment and floor sizes, function, and industry. Establishments can play multiple roles, being able to behave as a receiver (or consumer), a shipper (or supplier), and a carrier (or a third-party logistics service provider). Commodity contracts and logistics planning are associated with establishmentlevel decisions. As for the application in Singapore, the synthetic population of establishments was developed based on various business statistics (Le et al. 2016).

\subsubsection{Commodity Contract Estimation (LT Model)}

Commodity contracts define selling and purchasing policies and are the basis of the commodity flows between establishments. Each commodity contract specifies shipper and receiver locations, commodity type, amount of goods, and shipment size and frequency. The commodity contract estimation is composed of three separate steps: (1) freight generation, (2) shipper selection, and (3) size and frequency choice (Fig. 12.7). Freight generation starts with identifying whether each establishment is a shipper or receiver, using a logit model. Then, multinomial logit models simulate the selection of commodity types for outbound and inbound shipments. Finally,

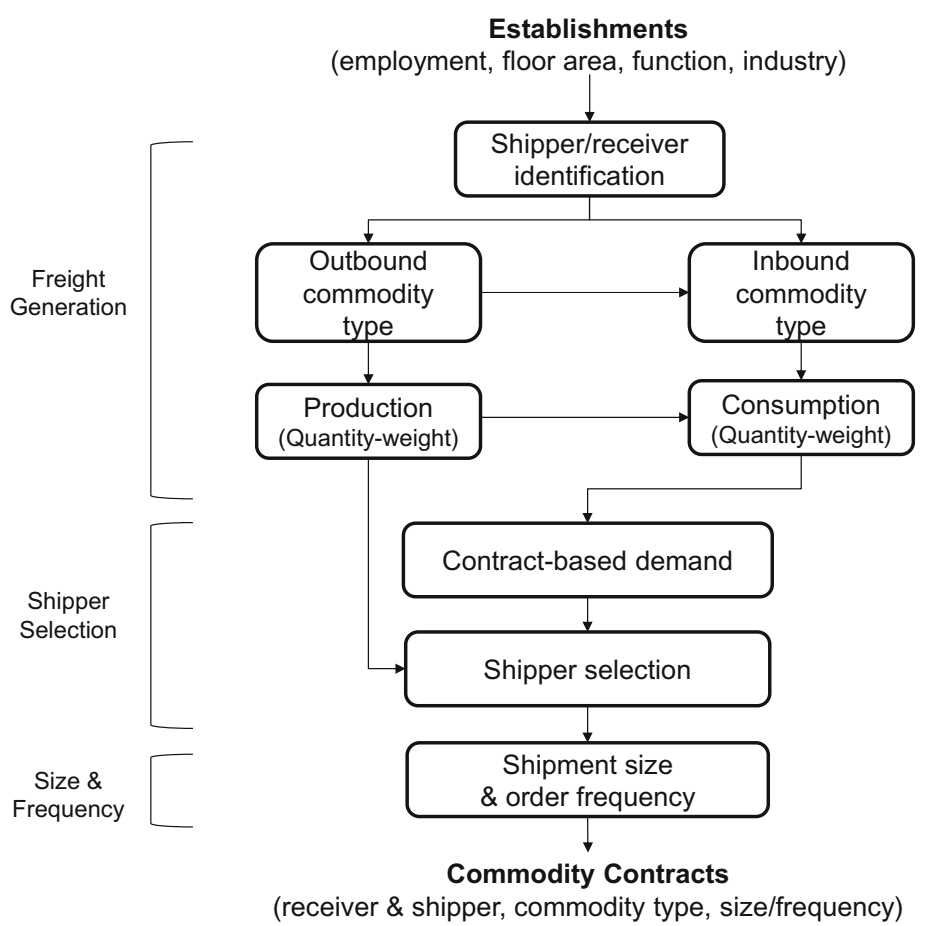

Fig. 12.7 Flow of the commodity contract estimation 
the quantities of production and consumption, which are quantities shipped and received, respectively, for a certain time period, are determined using linear models. In the following step-shipper selection-the estimated consumptions are used to generate contract-based demands. Each contract-based demand requires a single shipper (supplier), and each contract is made for a single receiver-shipper pair. A receiver can make one or more contracts with shippers. Logit mixture models with error components simulate shipper selection, considering the correlations among the alternative shippers with the same distribution channel type (Sakai et al. 2018). In the third step, linear models estimate shipment size and order frequency based on factors associated with the volume of goods, and transportation and inventory costs.

\subsubsection{Overnight Parking Choice (LT Model)}

Overnight parking choice is considered a long-term decision. We simulate the decisions of vehicle owners to assign parking lots for freight vehicles using multinomial logit models, using freight-vehicle population and overnight parking supply for freight vehicles as inputs. This module enables the simulations to evaluate the impacts of parking supply policies and to define their starting and end point of daily trips.

\subsubsection{Pre-day Logistics Planning (MT Model)}

Logistics planning processes convert shipment demand into vehicle-operation plans (VOPs). The VOPs define trips or tours of vehicles to be performed in a given day, including details about stop locations and the purposes (e.g., delivery of a specific shipment) and duration of stops. The logistics planning process has sub-modules for carrier selection and vehicle-operation planning, both of which are rule-based. A carrier is assigned to each shipment based on the distances from the shipment origin to potential carriers (i.e., transportation service providers), subject to their transport capacities. Vehicle-operation planning simulates the process of assigning shipments to vehicles as well as determining the orders of pickups and deliveries. In this submodule, a custom algorithm is applied to consolidate shipments and estimate stop duration for pickups and deliveries in a realistic manner.

\subsubsection{Within-Day Vehicle Operations (MT Model)}

VOPs are used as inputs for simulating vehicle operations and network traffic within a given day. Multinomial logit models simulate route choices for trips (i.e., movements from one location to another) based on route attributes, and driver and vehicle characteristics. Furthermore, another set of multinomial logit models simulates pickup and delivery parking choices considering cost, capacity, and congestion of parking facilities near the stop points (i.e., the activity locations), subject to parking-infrastructure 
data availability. A mesoscopic traffic simulation is run jointly with these simulations while updating network conditions.

\subsubsection{Visualization of Outputs}

The freight simulator runs at a metropolitan scale, which allows the measurement of the impacts of policies, technologies, or other system-related changes. Figures 12.8 and 12.9 show the examples of outputs from the LT and MT models, respectively. It should be noted that these figures are made only for illustrative purposes using a test data set and are not representative of the predicted flows. Figure 12.8 covers industryto-industry and zone-to-zone commodity flows and overnight parking locations of freight vehicles. Figure 12.9 includes delivery locations by freight vehicles, durations of vehicle usage in VOPs, and network traffic volume.

\subsubsection{Applications}

SimMobility supports the evaluation of a wide range of policies, from long-term landuse development plans to short-term parking-infrastructure operations. A series of urban freight case studies have been conducted for policy analysis purposes, with others being designed, including:

- Land-use changes, specifically those related to new industrial development as well as regulatory policies designed to mitigate negative impacts;

- Overnight parking-infrastructure supply policies (Gopalakrishnan et al. 2019);

- Urban consolidation policies involving participation by shippers, carriers, and receivers;

- Regulations to promote off-hour deliveries; and

- Route restrictions for goods vehicles.

\subsection{Demonstrations}

The two laboratories have been used jointly to advance the state of the art in behavioral modeling and simulation. We provide three cases demonstrating such joint use, focusing on their complementarity rather than the applications of the tool for decisionmaking processes, which is the subject of other publications (e.g., Gopalakrishnan et al. 2019).

The first case is the estimation of freight route-choice models, the second is the quantification of the performance of freight models (applied to vehicle tour formation models), and the third is the replication of freight and non-freight-vehicle tours for specific vehicular operation patterns that are not captured by conventional 
12 Laboratories for Research on Freight Systems and Planning
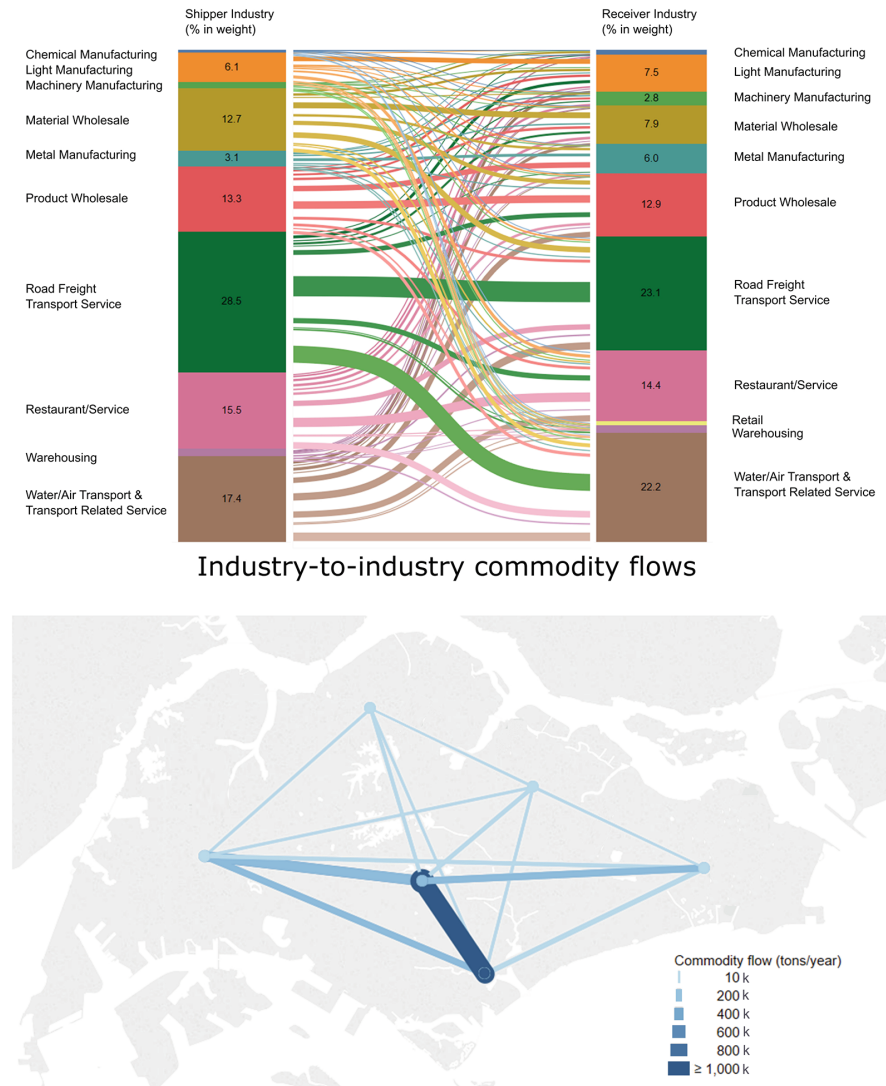

Zone-to-zone commodity flows

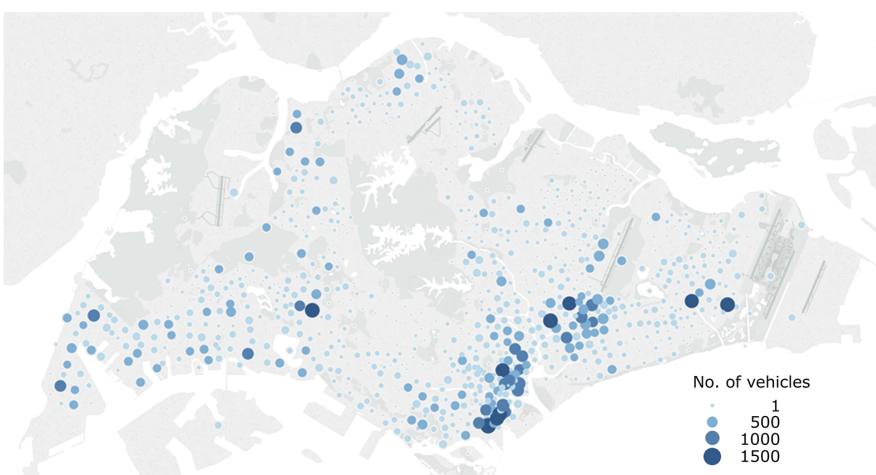

Overnight parking locations

Fig. 12.8 Illustrative outputs from the long-term model 

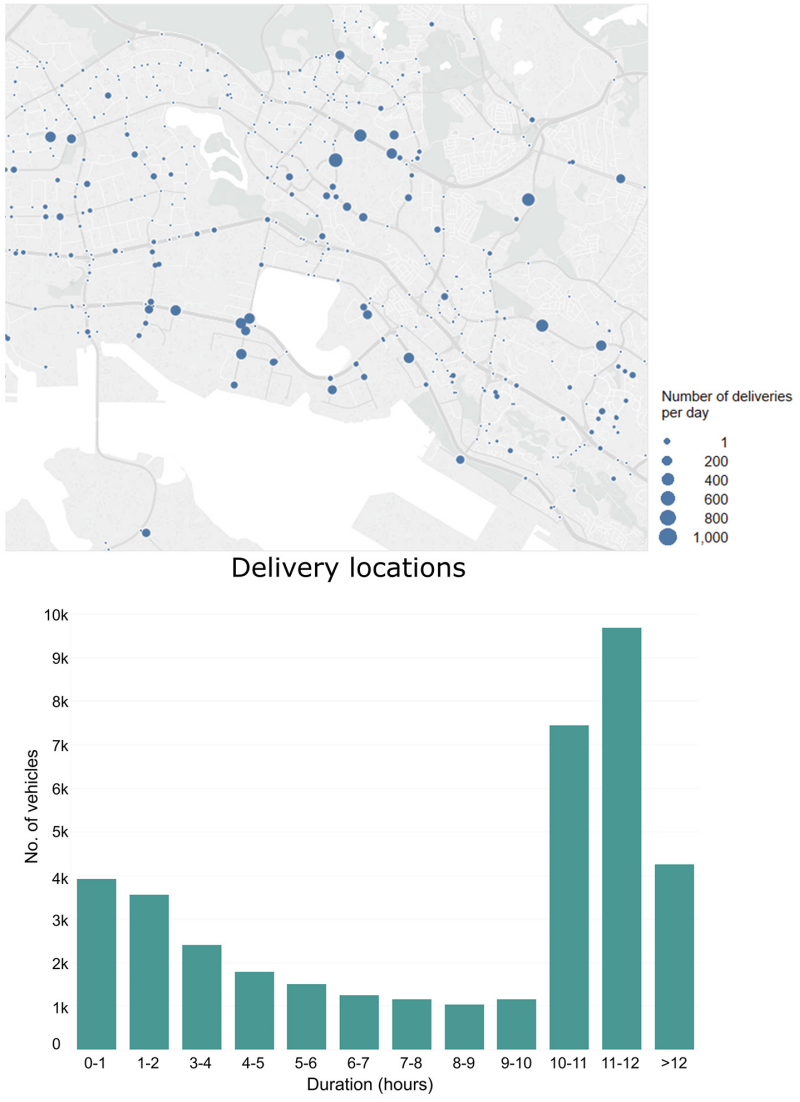

Duration of vehicle operation

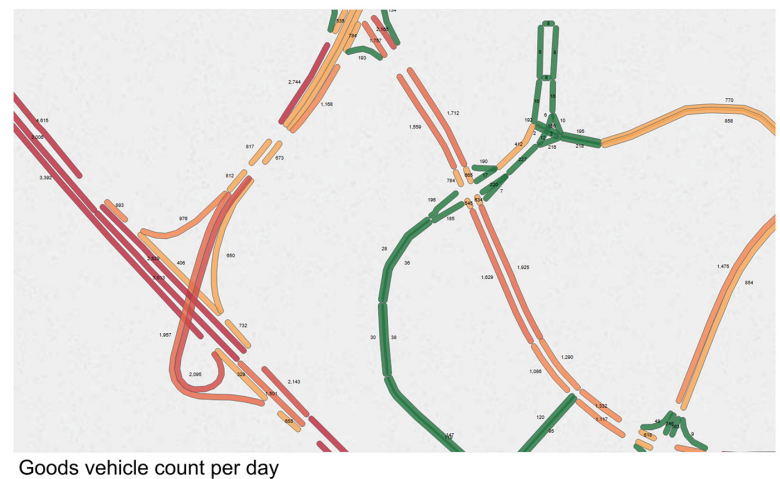

5000

Network traffic volume

Fig. 12.9 Illustrative outputs from the mid-term model 
demand models. More details about these applications can be found in the following references: Toledo et al. (2018), Alho et al. (2019b), and Gopalakrishnan et al. (2019).

\subsubsection{Freight-Vehicle Route-Choice Model}

The first application is the estimation of a freight-vehicle route-choice model. The route-choice decision of freight-vehicle drivers differs from that of passenger-vehicle drivers in terms of higher sensitivity to traffic conditions, and greater heterogeneity among driver types and associated commodity attributes, among other factors. The first step was to develop a truck-driver survey using FMS-Freight, which was conducted in the USA (Ben-Akiva et al. 2016). The survey collected user-annotated GPS data and characteristics of operational practices, vehicles, and drivers. A multinomial logit model was estimated using the dataset and applied to simulate the withinday route choice of drivers in SimMobility using the mid-term model. Explanatory variables include (1) traffic network attributes, which are generated by the supply simulation (e.g., travel time) or stored in the SimMobility database (e.g., road class, distance); and (2) characteristics of the driver and the vehicle, which are generated in the SimMobility long-term model. The model takes the value of explanatory variables as inputs and predicts the route between a given set of OD pairs with a Monte Carlo procedure. Figure 12.10 illustrates how the data collected using FMS-Freight are used to develop a freight route-choice model and how the model is applied in SimMobility.

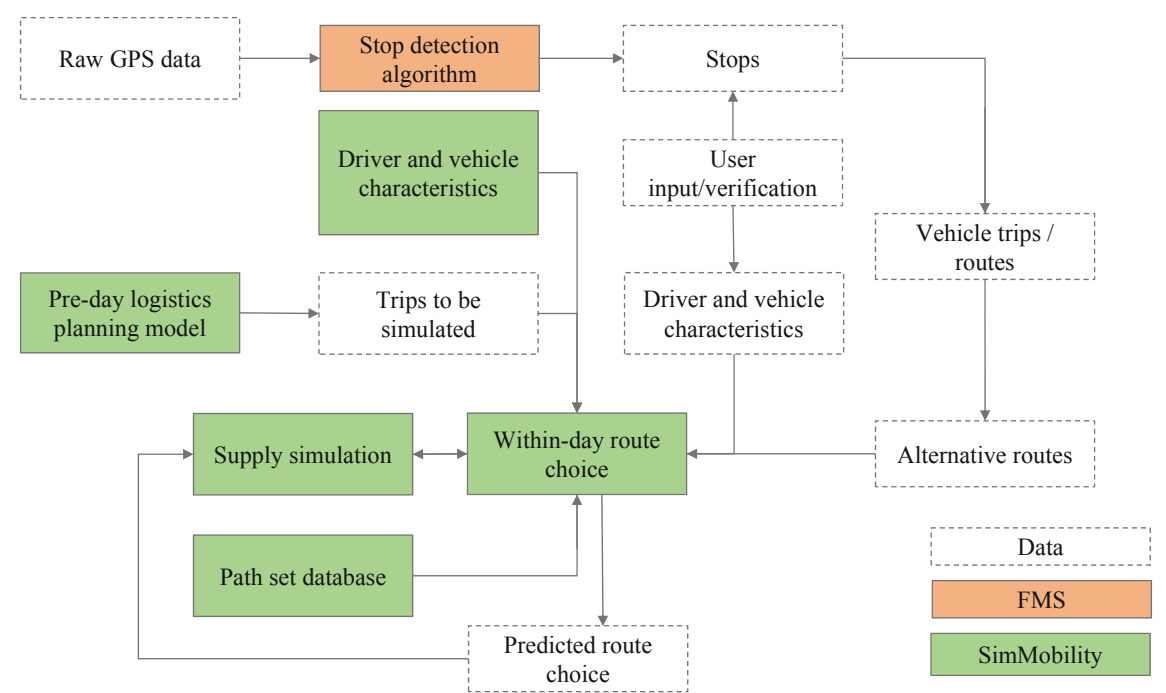

Fig. 12.10 Data and model flow for freight-vehicle route choice 


\subsubsection{Quantification of Model Performance}

The second case is the application of the laboratories to explore the research question: What is the value of using additional data and more sophisticated model formulations? We targeted the research question specifically at vehicle-operation planning, which generates tours in the freight simulator, and used data collected using FMS-Freight to compare the model formulations' outputs against observed truck flows. We evaluate discrepancies in zone-to-zone flows, realizing that some of the proposed methods applied in SimMobility achieve superior performance against state-of-the-practice methods. The process of integrating the data between both laboratories is summarized in Fig. 12.11. In broad terms, verified vehicle stops are associated with specific vehicle tours. Further details on the algorithms that can be used for this purpose can be found in papers by Alho et al. (2019a, b). Once tours are identified, specific tour-types allow commodity flows to be estimated (Alho et al. 2018). These commodity flows are used as an input to the SimMobility mid-term logistics planning model. By varying the formulation of this model, different vehicle OD flows are generated, which can then be compared with the original OD flows revealed from the data to assess model performance in replicating such flows.

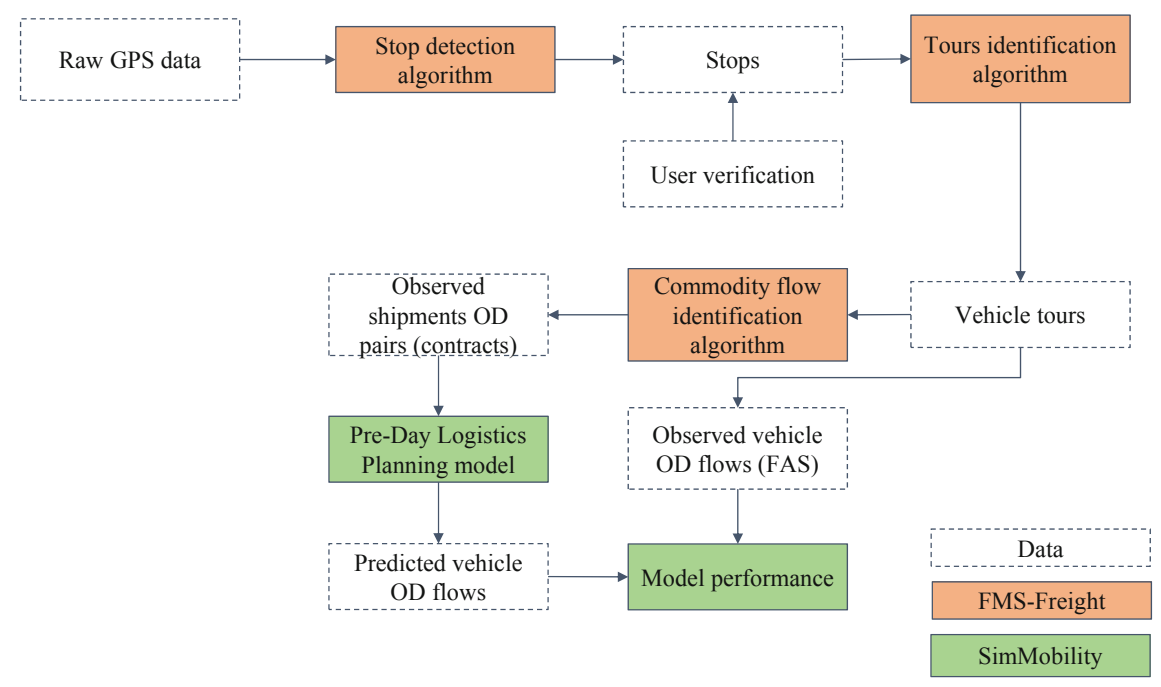

Fig. 12.11 Data and model flow for model performance quantification 


\subsubsection{Replication of Specific Freight and Non-Freight-Vehicle Tours}

The final selected application is related to the replication of specific freight and nonfreight-vehicle tours. The research team has performed a case-study in Singapore where simulation was used to assess a hypothetical scenario of overnight parkinginfrastructure re-organization, and associated tours performance. If the overnight parking infrastructure and the assignment of vehicles to it are optimized, this can contribute to reducing empty travel, and reducing traffic congestion and air pollution. For this purpose, vehicle trips to and from overnight parking locations had to be replicated. Since the overnight parking lots are not only occupied by conventional freight vehicles, but also by private buses (on-demand, for use by companies, tourism, among other uses) and service vehicles (e.g., some construction vehicles such as cranes), there was a need to replicate the tours of both these vehicle and operation types. It should be noted that demand models for these vehicle and operation types are commonly estimated as OD matrices and not at a level of detail we required for our simulations. Thus, the approach illustrated in Fig. 12.12 was applied. This required expanding the sampled tours to the relevant vehicle populations of subscribers of the overnight parking lots.

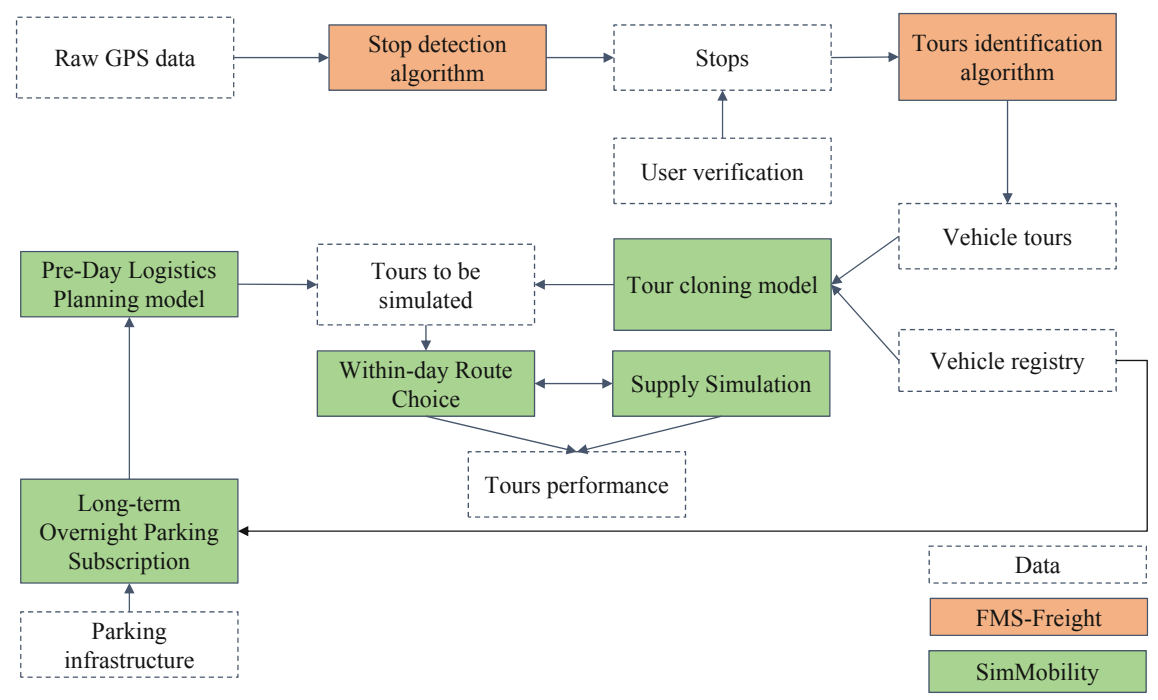

Fig. 12.12 Data and model flow for sample replication of tours of specific vehicle types 


\subsection{Concluding Remarks}

Urban freight data-collection and modeling techniques are currently portrayed at a transition point. Meersman and Van de Voorde (2019) question whether past and current data-collection methods are suitable to inform current and future modeling needs. For all we know, the evolution of methods is predominantly incremental. We put forward that laboratories such as those demonstrated in this chapter are key to the assessment of new approaches to data collection and modeling, including a quantitative assessment of the alternative's performance against the prior. Furthermore, we demonstrate that the research progress in either data collection, or modeling and simulation, can be augmented by coordinated use of their capabilities.

The pace of change in urban freight transport appears to grow faster, and with critical implications to the relevance of freight models in assessing technological and policy impacts. This calls for further attention to the representation of relevant agents in the urban freight system in simulations, as well as their behaviors and interactions. For the latter cases, the role of sensing technologies is key to reducing survey fatigue and allowing for lengthier and deeper data-collection efforts.

Acknowledgements This research is supported in part by the Singapore Ministry of National Development and the National Research Foundation, Prime Minister's Office, under the Land and Liveability National Innovation Challenge (L2 NIC) Research Programme (L2 NIC Award No L2 NICTDF1-2016-1). Any opinions, findings, and conclusions or recommendations expressed in this material are those of the author(s) only and do not reflect the views of the Singapore Ministry of National Development and National Research Foundation, Prime Minister's Office, Singapore. We thank the Urban Redevelopment Authority of Singapore, JTC Corporation, Land Transport Authority of Singapore and Housing and Development Board of Singapore for their support.

\section{References}

Adnan M, Francisco CP, Carlos LA, Kakali B, Milan L, Sebastian R, Yi Z, Joseph F, Christopher Z, Moshe B (2016) SimMobility: a multi-scale integrated agent-based simulation platform. Paper presented at the 95th annual meeting of the transportation research board, Washington

Alho AR, de Abreu e Silva J (2015) Lisbon's establishment-based freight survey: revealing retail establishments' characteristics, goods ordering and delivery processes. Eur Transp Res Rev 7:16. https://doi.org/10.1007/s12544-015-0163-7

Alho AR, You L, Lu F, Lynette C, Fang Z, Moshe B (2018) Next-generation freight vehicle surveys by supplementing truck GPS tracking with a driver activity survey: account and insights. In: 21 st international conference on intelligent transportation systems (ITSC), Maui, 4-7 Nov 2018, pp 2974-2979. https://doi.org/10.1109/ITSC.2018.8569747

Alho AR, Sakai T, Chua MH, Jeong K, Jing P, Ben-Akiva M (2019a) Exploring methods for revealing freight vehicle tours, tour-types and tour-chains from GPS vehicle tracking and driver survey data. J Big Data Anal Transp

Alho AR, Sakai T, Chua MH, Raven M, Hara Y, Ben-Akiva M (2019b) The influence of tourformation assumptions on the reproducibility of freight vehicle flows. In: Proceedings of the eleventh city logistics conference, Dubrovnik 
Allen J, Browne M, Cherrett T (2012) Survey techniques in urban freight transport studies. Transp Rev Transnatl Transdiscipl J 32(3):287-311. https://doi.org/10.1080/01441647.2012.665949

Azevedo CL, Deshmukh NM, Marimuthu B, Oh S, Marczuk K, Soh H, Basak K, Toledo T, Peh L, Ben-Akiva M (2017) SimMobility short-term: an integrated microscopic mobility simulator. Transp Res Rec 2622:13-23

Ben-Akiva ME, Toledo T, Santos J, Cox N, Zhao F, Lee YJ, Marzano V (2016) Freight data collection using GPS and web-based surveys: insights from US truck drivers' survey and perspectives for urban freight. Case Stud Transp Policy 4(1):38-44. https://doi.org/10.1016/j.cstp.2015.11.005

Boerkamps J, van Binsbergen A, Bovy P (2000) Modeling behavioral aspects of urban freight movement in supply chains. Transp Res Rec 1725:17-25

Cheah L, Zhao F, Stinson M, Lu FP, Dingmastera J, Marzano V, Ben-Akiva M (2017) Nextgeneration commodity flow survey: a pilot in Singapore. Paper presented at city logistics conference, Phuket

Chow JY, Yang CH, Regan AC (2010) State-of-the art of freight forecast modeling: lessons learned and the road ahead. Transportation 37(6):1011-1030

Eluru N, Li X, Pinjari A, Abdelaty M, Anowar S, Momtaz SU, Iraganaboina NC, Keya N, Dey B, Zhao D, Balusu SPK, Sheela PV (2018) Freight data fusion from multiple data sources for freight planning applications in Florida. Final report. University of Central Florida, Department of Civil, Environmental and Construction Engineering

Fischer M, Outwatern M, Cheng L, Ahanotu DN, Calix R (2005) Innovative framework for modeling freight transportation in Los Angeles County, California. Transp Res Rec 1906:105-112

Gopalakrishnan R, Alho AR, Sakai T, Cheah L, Ben-Akiva M (2019) Assessing overnight parking infrastructure policies for commercial vehicles in cities using agent-based simulation. In: Proceedings of the eleventh city logistics conference, Dubrovnik

Holguín-Veras J, Jaller M (2013) Comprehensive freight demand data collection framework for large urban areas. In: Gonzalez-Feliu J et al (eds) Sustainable urban logistics: concepts, methods and information systems. EcoProduction. Springer-Verlag, Berlin Heidelberg, pp 91-112. https:// doi.org/10.1007/978-3-642-31788-0_6

Jeong K, Tok A, Ritchie SG, Park J (2016) California vehicle inventory and use survey: pilot study insights. Transp Res Rec 2547(1):32-40. https://doi.org/10.3141/2547-06

Kamali M (2015) Development of truck route choice data using truck GPS. M.Sc. thesis, University of South Florida. https://scholarcommons.usf.edu/cgi/viewcontent.cgi?referer=https://www.goo gle.com/\&httpsredir=1\&article=7164\&context=etd. Accessed 27 Feb 2019

Le DT, Cernicchiaro G, Zegras C et al (2016) Constructing a synthetic population of establishments for the simmobility microsimulation platform. Transp Res Procedia 19:81-93. https://doi.org/10. 1016/j.trpro.2016.12.070

Livshits V, You D, Zhu H, Jeon K, Vallabhaneni L, Camargo P, Hong S, Nippani S, Sun A, Noh H, Tokishi J, Kuppam A, Selby B, Beagan D, Murray D, Smith D, Lemp J, Short J, Viswanathan K, Ehlen M, Stinson M, Khurana M, Bingham P, Bostrom R, Golub S, Carlin G, Ravulaparthy S, Pourabdollahi Z (2018) Mega-regional multi-modal agent-based behavioral freight model. Final report. Strategic highway research program 2 (SHRP2 C20 IAP grant). https://www.azmag.gov/Portals/0/Documents/MagContent/TRANS_2017-02-13_ SHRP2-TRANS_2017-06-06-C20-MAG-Next-Generation-Freight-Demand-Model-Update.pdf

Lu Y, Adnan M, Basak K, Pereira FC (2015) SimMobility mid-term simulator: a state of the art integrated agent based demand and supply model. In: 94th annual meeting of the transportation research board, Washington

Ludlow D, Sakhrani V (2017) NCFRP 49-new source of freight data for urban and metropolitan mobility. In: 2017 international urban freight conference (I-NUF), Long Beach. https://www.met rans.org/sites/default/files/Special\%20Session\%201\%20Ludlow\%20.pdf. Accessed 27 Feb 2019

Mammes N, Klatsky M (2017) Approaches to monitor truck loading activity in New York City using video analytics. In: TRB innovations in freight data workshop, Irvine, 18 May 2017. https://online pubs.trb.org/onlinepubs/conferences/2017/FreightData/MammesPPT.pdf. Accessed 27 Feb 2019 
Meersman H, Van de Voorde E (2019) Freight transport models: ready to support transport policy of the future? Transp Policy 83:97-101. https://doi.org/10.1016/j.tranpol.2019.01.014

Nahmias-Biran B, Han Y, Bekhor S, Zhao F, Zegras C, Ben-Akiva M (2018) Enriching activity-based models using smartphone-based travel surveys. Transp Res Rec 2672(42):280-291

Outwater M, Smith C, Wies K et al (2013) Tour based and supply chain modeling for freight: integrated model demonstration in Chicago. Transp Lett 2:55-66

Resource Systems Group, Inc (2015) User's guide and model documentation: agent-based supply chain modeling tool. The report for Chicago metropolitan agency for planning. https://www. cmap.illinois.gov/data/transportation/modeling

Roorda MJ, Cavalcante R, McCabe S, Kwan H (2010) A conceptual framework for agent-based modelling of logistics services. Transp Res Part E 46(1):18-31

Sakai T, Bhavathrathan BK, Alho AR, Hyodo T, Ben-Akiva M (2018) Commodity flow estimation for a metropolitan scale freight modeling system: supplier selection considering distribution channel using an error component logit mixture model. Transportation 1-29. https://doi.org/10. 1007/s11116-018-9932-1

Sakai T, Alho AR, Bhavathrathan BK, Dalla Chiara G, Gopalakrishnan R, Jing P, Hyodo T, Cheah L, Ben-Akiva M (2019) SimMobility freight: an agent-based urban freight simulator for evaluating logistics solutions. Transp Res Part E Logist Transp Rev

Sun X, Ding J, Dalla Chiara G, Cheah L, Cheung NM (2017) A generic framework for monitoring local freight traffic movements using computer vision-based techniques In: 5th IEEE international conference on models and technologies for intelligent transportation systems (MT-ITS), Naples, pp 63-68. https://ieeexplore.ieee.org/abstract/document/8005592

Tok YC (2008) Commercial vehicle classification system using advanced inductive loop technology. UC Berkeley: University of California Transportation Center. Retrieved from https://escholars hip.org/uc/item/92x23786

Toledo T, Jing P, Atasoy B, Ding Mastera J, Santos JO, Ben-Akiva M (2018) Intercity truck driver route choice incorporating drivers' heterogeneity in toll road usage: data collection, model estimation, and model application. In: Transportation research board 97th annual meeting, Washington

Wisetjindawat W, Sano K, Matsumoto S (2005) Supply chain simulation for modeling the interactions in freight movement. J East Asian Soc Transp Stud 6:2991-3004

You L, Zhao F, Cheah L, Jeong K, Zegras C, Ben-Akiva M (2018) Future mobility sensing: an intelligent mobility data collection and visualization platform. In: 21st IEEE international conference on intelligent transportation systems, Maui, 4-7 Nov

Zanjani AB (2014) Deriving statewide freight truck flows from global positioning system (GPS) data. M.Sc. dissertation, University of South Florida. https://scholarcommons.usf.edu/cgi/ viewcontent.cgi?referer=https://www.google.com/\&httpsredir=1\&article=6373\&context=etd. Accessed 17 Feb 2019

Zhang G, Avery RP, Wang Y (2007) A video-based vehicle detection and classification system for real-time traffic data collection using uncalibrated video cameras. Transp Res Rec 1993(1):138147. https://doi.org/10.3141/1993-19

Zhao F, Ghorpade A, Pereira FC, Zegras C, Ben-Akiva M (2015) Stop detection in smartphone-based travel surveys. Transp Res Procedia 11:218-226

Zhu Y, Diao M, Ferreira J Jr, Zegras PC (2018) An integrated microsimulation approach to land-use and mobility modeling. J Transp Land Use 11(1):633-659 

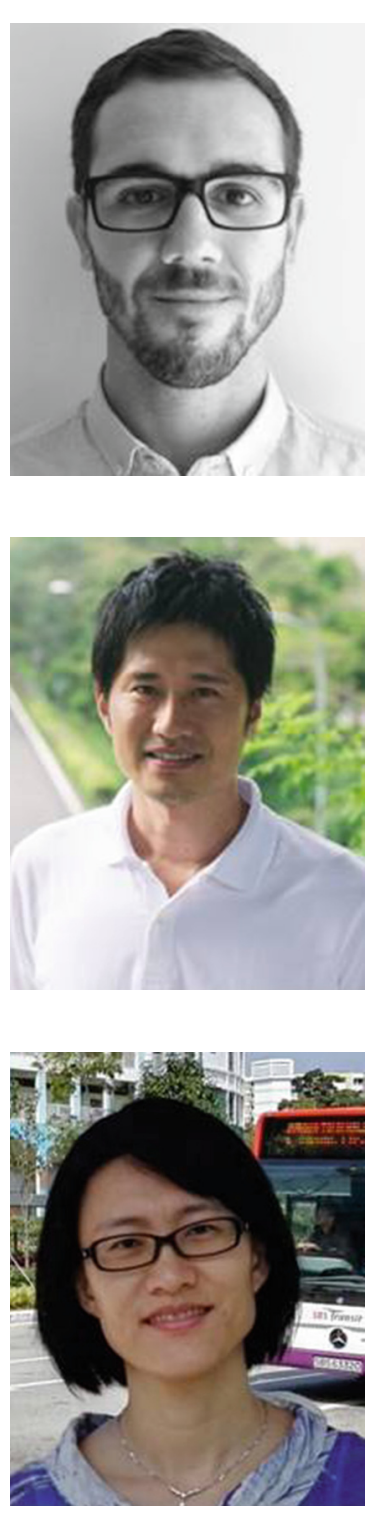

André Romano Alho is a Research Scientist in the Future Urban Mobility Interdisciplinary Research Group, at the Singapore-MIT Alliance for Research and Technology. He is passionate about developing and applying research methods to provide innovative solutions for Transportation Systems, particularly focusing on Urban Freight operations.

Takanori Sakai is a Senior Postdoctoral Associate in the Future Urban Mobility Interdisciplinary Research Group at the Singapore-MIT Alliance for Research and Technology. His research mainly focuses on urban freight transportation planning and modeling and transportation geography. He is a member of the Transportation Research Board-Freight Transportation Planning and Logistics Committee.

Fang Zhao is a Research Scientist at the Singapore-MIT Alliance for Research and Technology, Future Urban Mobility Interdisciplinary Research Group. She has an inter-disciplinary background with expertise in machine learning, communication networks, and travel surveys. She received her Ph.D. degree in Electrical Engineering from the Massachusetts Institute of Technology. 

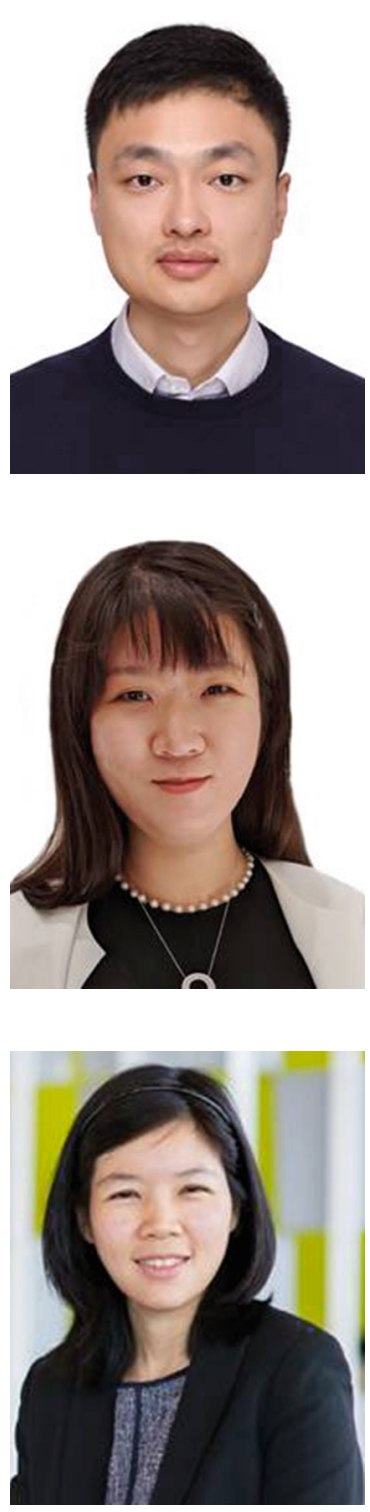

Linlin You is an Associate Professor at the School of Intelligent Systems Engineering, Sun Yat-sen University, and also a Research Affiliate at the Intelligent Transportation Systems Lab, Massachusetts Institute of Technology. He is a member of IEEE and ACM, and interested in Smart Service Orchestration, ITS and Multi-source data fusion.

Peiyu Jing is a Transportation Ph.D. candidate in the Department of Civil and Environmental Engineering at Massachusetts Institute of Technology. Her research interest is freight data collection, agent-based freight modeling and simulation, and freight congestion pricing.

Lynette Cheah is an Associate Professor of Engineering Systems at the Singapore University of Technology and Design. She leads the Sustainable Urban Mobility research group, which develops data-driven models and tools to reduce the environmental impacts of passenger and urban freight transport. 


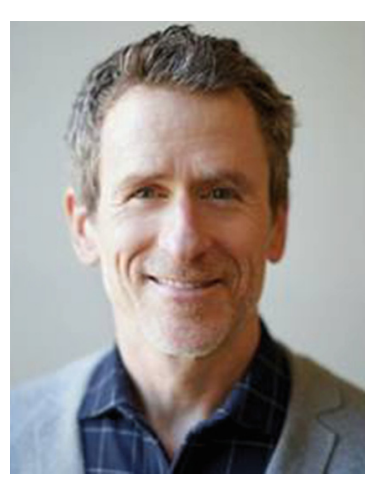

Christopher Zegras is Professor of Mobility and Urban Planning at the Massachusetts Institute of Technology where he is Head of the Department of Urban Studies and Planning. He is also a Principal Investigator of the Future of Urban Mobility Interdisciplinary Research Group, under the Singapore MIT Alliance for Research and Technology.

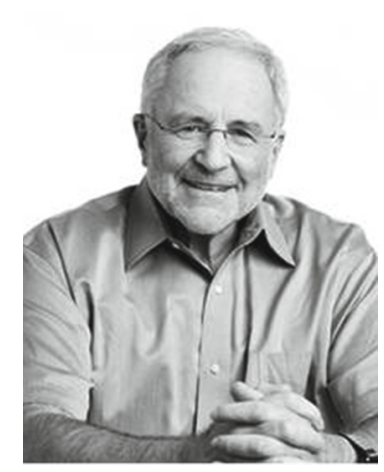

Moshe Ben-Akiva is the Edmund K. Turner Professor of Civil and Environmental Engineering at Massachusetts Institute of Technology (MIT), Director of MIT's Intelligent Transportation Systems Lab, and Principal Investigator at the Singapore-MIT Alliance for Research and Technology. His interests include Smart Mobility and discrete choice analysis with machine learning capabilities.

Open Access This chapter is licensed under the terms of the Creative Commons Attribution 4.0 International License (http://creativecommons.org/licenses/by/4.0/), which permits use, sharing, adaptation, distribution and reproduction in any medium or format, as long as you give appropriate credit to the original author(s) and the source, provide a link to the Creative Commons license and indicate if changes were made.

The images or other third party material in this chapter are included in the chapter's Creative Commons license, unless indicated otherwise in a credit line to the material. If material is not included in the chapter's Creative Commons license and your intended use is not permitted by statutory regulation or exceeds the permitted use, you will need to obtain permission directly from the copyright holder.

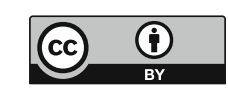

